\title{
Introduction to the special issue on software defined radio: selected papers from the Wireless Innovations Forum's SDR'13-WinnComm
}

\author{
John Glossner • Mohammed Ismail • \\ Jarmo Takala
}

Published online: 6 December 2013

(C) Springer Science+Business Media New York 2013

This special issue contains extended articles of the selected papers from the research and development (R\&D) track of the wireless innovation Forum's SDR'13-WinnComm conference on wireless communications technologies and software defined radio.

Established in 1996, the Wireless Innovation Forum ${ }^{\text {TM }}$ is an non-profit "mutual benefit corporation" dedicated to advocating for the innovative use of spectrum, and advancing radio technologies that support essential or critical communications worldwide. Forum members bring a broad base of experience in software defined radio (SDR), cognitive radio (CR) and dynamic spectrum access (DSA) technologies in diverse markets and at all levels of the wireless value chain to address emerging wireless communications requirements through enhanced value, reduced total life cost of ownership, and accelerated deployment of standardized families of products, technologies, and services. The Forum acts as the premier venue for its members to collaborate to achieve these objectives, providing opportunities to network with customers, partners and competitors, educate decision makers, develop and expand markets and advance relevant technologies.

\footnotetext{
J. Glossner

Optimum Semiconductor Technologies Inc., 120 White Plains

Rd., Tarrytown, NY 10591, USA

e-mail: jglossner@optimumsemi.com

M. Ismail

Khalifa University of Science Technology and Research

(KUSTAR), Sharjah, Abu Dhabi, UAE

e-mail: ismail@kustar.ac.ae

J. Takala $(\bowtie)$

Department of Pervasive Computing, Tampere University of

Technology, Korkeakoulunkatu 1, 33720 Tampere, Finland

e-mail: jarmo.takala@tut.fi
}

The Technical Conference was first established in 2002 after a highly successful workshop held in 2001. Now attracting a broad range of delegates from over 20 different countries, the conference is the only event devoted to the advancement of reconfigurable radio technologies from research through deployment.

A unique feature of this conference is in providing opportunities to network from a broad mix of participants from all levels of the wireless value chain including investors, commercial network operators, radio manufacturers, system integrators, government procurement officials, regulators, technology providers, engineering service providers, and consultants. The academically focused papers are submitted to the R\&D track of the conference. This year's paper topics included software defined radio implementations and architectures, communications signal processing, physical layer techniques, chip implementations, GPUs, FPGAs, processors, RF technologies, security, software systems, software communication architecture, spectrum sharing, cognitive radio, system implementation, and testing.

This special issue specifically highlights the selected R\&D papers from the 2013 SDR'13-WinnComm conference. The extended papers were evaluated by world experts in software defined radio, cognitive radio, dynamic spectrum access, and communications system design.

This special issue contains 18 articles. A network simulation model framework for SDR is discussed by Sapello, Serban, Sethi, Chiang, and Moeltner. The framework takes into account time delays in the SDR processes and supports reconfiguration. The accuracy and scalability are evaluated in the paper. A hardware-accelerated design space exploration framework for communication systems is proposed by Kock, Hasselbarth, Pfitzner, and Blume. The framework combines high-level tools with system-on-chip template 
mapped on an FPGA-based emulation system. The proposed framework is illustrated and verified with two case studies. Ozgul, Langer, Noguera, and Vissers present a design flow supporting partitioning on hardware and software components and compare different techniques for integrating such components. The authors use digital pre-distortion as an example and compare area/latency cost trade-offs and discuss energy savings on FPGA fabric.

DVB-T2 constellation demapping on GPUs is considered by Grönroos, Nybom, and Björkqvist as they investigate simplifications for demapping process for rotated QAM constellations. The experiments show that simplified demapper and LDPC decoder can share be same GPU. Bang, Ahn, Jin, Choi, Glossner, and Ahn describe an implementation of LTE system on an SDR Platform containing a GPU. The parallel resources of GPU are exploited in particular on turbo decoding. Experiments show that the implementation fulfills realtime requirements. Design of a vector processor for turbo decoding is discussed by Shahabuddin, Janhunen, Juntti, Ghazi, and Silvén. The authors have used the transport triggered architecture as a processor template and report the performance of a processor customized for max-logMAP algorithm and synthesized on $90 \mathrm{~nm}$ IC technology.

A comparison of LTE channelizers is provided by Harris, Dick, Venosa, and Chen who analyze channelizers and show that recursive filters provide lower arithmetic complexity and group delay than FIR filters. Harris, Venosa, Chen, and Dick present a novel application of perfect reconstruction filter banks in 3GPP LTE and UMTS Radios. The analysis-synthesis chains have been optimized to maximize the performance with low arithmetic complexity in given applications. Detector implementations for 3G LTE downlink and uplink are discussed by Hänninen, Janhunen, and Juntti. They study programmable and configurable detector and QR decomposition implementations where the downlink transmission uses OFDM and the implementation is based on transport triggered architecture while uplink uses singlecarrier FDMA and is implemented on FPGA.

Xie and Bose present an improvement for partial update Euclidean direction search algorithm for adaptive filtering. The proposed method provides significant reduction in computational complexity compared to full update methods. The steady-state performance has been verified with simulations and theoretical studies. Sinha, Balamuralidhar, and Bhujade propose a data communi- cation on conventional audio interface of mobile devices by exploiting the frequency range of $16-20 \mathrm{kHz}$. The authors have built a prototype to show the potential of the proposed method.

Ankaralı, Şahin, and Arslan propose improving spectral efficiency of multicarrier schemes by allowing controlled overlapping of neighboring symbols in time and frequency. The method exploits user-specific filters, which have different tie-frequency characteristics. Coding-based ultrawideband digital beamformer is discussed by Alwan, Balasubramanian, Atallah, LaRue, Sertel, Khalil, and Volakis. They propose a novel transceiver architecture for cognitive sensing, which uses onsite coding. The proposed solution reduces significantly the number of $\mathrm{AD}$ converters and $\mathrm{I} / \mathrm{O}$ pins. Imana, Reed, and Yang propose a mechanism to control local oscillator and sampling frequencies in a receiver RF front-end to relax pre-selector filter selectivity requirements. The method allows improved reliability for receivers and it is suitable for agile applications such as dynamic spectrum access.

Secondary devices operating on TV white spaces are discussed by Almeida, Chaves, Vieira, and Iida. They propose the power level of secondary devices to be defined based on device location obtained from geo-location databases. The paper proposes three different strategies, which all ensure required protection without significant implementation costs. Adrat, Osten, Leduc, Antweiler, and Elders-Boll suggest hierarchical modulation to be used to add enhanced waveforms on top of legacy waveforms. The proposed approach allows additional bit budget for increased data rate and/or robustness. Amorim, Vieira, and Carvalho propose a coordinated power control mechanism, which allows resource sharing under interference constraints. The paper shows two approaches to improve the spectrum efficiency with a cost of interference margin. Waveform reconstruction from ontological descriptions is considered by Lechowicz and Kokar. They propose a novel method, which allows radios with different hardware and software architectures, different software APIs or nonuniform waveform descriptions, to interoperate. The authors have built a proof-of-concept system, which confirms the feasibility of the method.

As guest editors of this special issue, we sincerely thank the authors for their valuable contributions and all the anonymous reviewers for their feedback and help in ensuring a high level of quality. We also extend our appreciation to S. Shenbagam for his help on setting up this issue. We hope that you enjoy the special issue and find the articles informative and useful. 


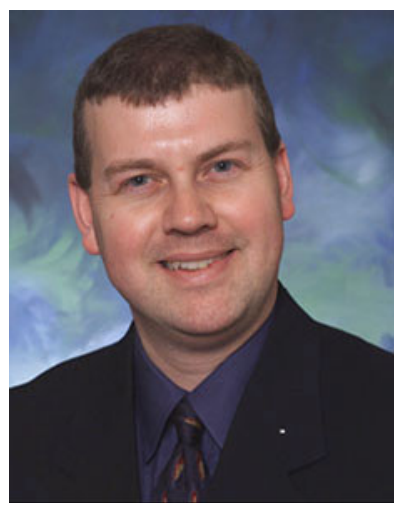

John Glossner is CEO of Optimum Semiconductor Technologies, the US division of Wuxi DSP. Prior to joining Wuxi DSP John co-founded Sandbridge Technologies and served as EVP \& CTO. Prior to Sandbridge, John managed both technical and business activities in DSP and Broadband Communications at IBM and Lucent/ Starcore. John received a Ph.D. in Electrical and Computer Engineering from TU Delft in the Netherlands, M.S degrees in E.E. and Eng. Mgt from NTU, and a B.S.E.E. degree from Penn State. John is an Officer, member of the Board of Directors and CTO of the Wireless Innovation Forum. He has more than 120 publications and 36 issued patents.

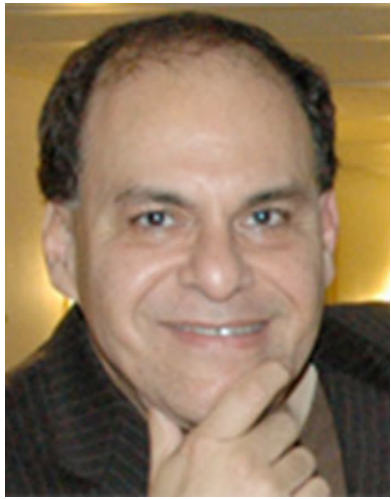

Mohammed Ismail a prolific author, professor and entrepreneur Joined Khalifa University of Science, Technology and Research (KUSTAR), UAE in April 2011. He spent over 20 years in academia and industry in the US and Europe and is the Founder of the Ohio State University's (OSU) Ana$\log$ VLSI Lab, one of the foremost research entities in the field of analog, mixed signal and RF integrated circuits. He also served on the Faculty of

OSU's ElectroScience Lab. He held a Research Chair at the Swedish Royal Institute of Technology (KTH) where he founded the RaMSiS (Radio and Mixed Signal Integrated Systems) Research Group there. He had visiting appointments in Finland (Aalto university), Norway (NTH and University of Oslo), the Netherlands (Twente University) and Japan (Tokyo Institute of Technology). At KUSTAR, Dr. Ismail holds the ATIC Professor Chair and served as Director of KUSTAR's Sharjah Campus. He is the Founding Chair of the newly established ECE Department with 40 faculty and 500 students, 450 undergraduate and 50 graduate students pursuing M.S. and Ph.D. programs. Dr. Ismail has initiated the new KUSTAR's ICT Research Center encompassing 5 Research Labs conducting multidisciplinary research in information security, e-services and networks, multimedia communications and embedded mixed signal systems. He is the founding co-Director of the ACE4S ATIC-KUSTAR Semiconductor Research Center focusing on low power System on Chip (SoC) design, test and IP development targeting the strategic areas of energy efficient wireless chip sets, bio chips, self powered devices, and power management. The Research is aligned with the 2030 Abu Dhabi strategic plan calling for diversification of the economy beyond oil and gas and for promoting innovation, entrepreneurship and spinoffs in the semiconductor, energy, public health and ICT sectors among others. Dr. Ismail's current research focuses on robust low power RF and mm-wave ICs for wireless, bio and multimedia applications with a focus on manufacturable low cost high volume nanometer CMOS solutions for mobile and self-powered wearable and embedded devices. A more recent interest is on nanoscience aiming to develop devices in the sub $10 \mathrm{~nm}$ range based on nano Schottky contacts. He served as a Corporate Consultant to over 30 companies and is a CoFounder of Micrys Inc., Columbus, Ohio, Spirea AB, Stockholm, Firstpass Technologies Inc., Dublin, Ohio and ANACAD-Egypt (now part of Mentor Graphics). He advised the work of over $50 \mathrm{Ph} . \mathrm{D}$. students and of over 100 M.S. students. He authored or co-authored over a dozen books and over 150 journal publications and has 7 US patents. He is the Founding Editor of the Springer Journal of Analog Integrated Circuits and Signal Processing and serves as the Journal's Editor-in-Chief. He served the IEEE in many editorial and administrative capacities. He is the Founder of the IEEE International Conference on Electronics, Circuits and Systems (ICECS), the flagship Region 8 Conference of the IEEE Circuits and Systems Society. He is a Consulting Editor of the Springer Advanced Analog Book Series. He received the US Presidential Young Investigator Award, the Ohio State Lumley Research Award four times, in 1992, 1997, 2002 and 2007 and the US Semiconductor Research Corporation's Inventor Recognition Award twice. He is a Fellow of IEEE.

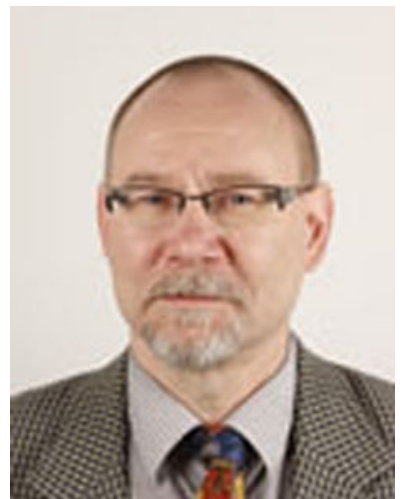

Jarmo Takala received his M.Sc. (hons) degree in Electrical Engineering and Dr. Tech. degree in Information Technology from Tampere University of Technology, Tampere, Finland (TUT) in 1987 and 1999, respectively. From 1992 to 1996, he was a Research Scientist at VTT-Automation, Tampere, Finland. Between 1995 and 1996, he was a Senior Research Engineer at Nokia Research Center, Tampere, Finland. From 1996 to 1999 , he was a Researcher at TUT. Currently, he is Professor on Computer Engineering at the Department of Pervasive Computing of TUT and Dean of the Faculty of Computing and Electrical Engineering of TUT. His research interests include parallel processing for digital signal processing systems both the hardware and software aspects. Dr. Takala is Co-Editor-in-Chief of Journal on Signal Processing Systems and he was Associate Editor for IEEE Transactions on Signal Processing Systems in 2007-2011. Dr. Takala was the Chair of IEEE Signal Processing Society Technical Committee on Design and Implementation of Signal Processing Systems during 2012-2013. Dr. Takala is a Senior Member of the IEEE. 